\title{
8 Interaktion von Temperatur und Luftschadstoffen: Einfluss auf Morbidität und Mortalität
}

\author{
Susanne Breitner, Regina Pickford und Alexandra Schneider \\ C. Günster | J. Klauber | B.P. Robra | C. Schmuker | A. Schneider (Hrsg.) Versorgungs-Report Klima und Gesundheit. \\ DOI 10.32745/9783954666270-8, @ MWV Medizinisch Wissenschaftliche Verlagsgesellschaft Berlin 2021
}

Zusammenhänge zwischen Luftschadstoffen und Gesundheit werden seit vielen Jahren erforscht. Obwohl die Luftschadstoffe primär über die Atemwege in den Körper gelangen, können sie auch Herz-Kreislauf-Erkrankungen auslösen. Luftverschmutzung und Klimawandel sind eng miteinander verknüpft. Trotzdem wurden gesundheitliche Effekte von Wetterveränderungen sowie Luftqualität lange Zeit isoliert betrachtet. Die potenziellen Pathomechanismen bei hoher Lufttemperatur legen im Vergleich zu den Mechanismen bei erhöhten Luftschadstoffkonzentrationen nahe, dass es bei vielen regulatorischen Abläufen im Körper Parallelen zwischen den Einflüssen der beiden Umweltfaktoren gibt. Somit ist vorstellbar, dass es hier zu Interaktionen und Synergien von Luftemperatur und Luftschadstoffen kommt. Das Zusammenspiel von Hitze und Luftschadstoffen macht es insgesamt schwerer, in der jeweiligen Situation jeweils die bestmöglichen präventiven Maßnahmen zu wählen - optimalerweise sollten sich Benefits für gleichzeitig bestehende Umweltrisiken ergeben. In Bezug auf die Einführung von Hitzewarnsystemen zeigt sich, dass weniger Menschen an übermäßiger Hitze starben und auch die Nachfrage nach Krankenwägen nach der Einführung dieser Systeme zurückging. Es besteht allerdings weiterhin Besorgnis darüber, ob die am stärksten gefährdeten Gruppen wie ältere Menschen und Obdachlose in diesen Maßnahmen angemessen erreicht werden können.

Associations between air pollutants and health have been investigated for many years. Although air pollutants enter the body primarily via the respiratory tract, they can also trigger cardiovascular diseases. Air pollution and climate change are closely linked. However, the health effects of weather changes and air quality were considered separately for a long time. The potential pathomechanisms related to high air temperature, in comparison to the mechanisms linked to elevated air pollutant concentrations, suggest that there are parallels between the influences of the two environmental factors in many regulatory processes in the body. Hence, it is conceivable that there will be interactions and synergies between air temperature and air pollutants. The interaction of heat and air pollutants makes it more challenging to choose the best possible preventive measures in the respective situation - optimally, there should be benefits for simultaneously existing environmental risks. Regarding the introduction of heat warning systems, fewer people have died from excessive heat, and the demand for ambulances has also decreased 
II Gesundheitliche Auswirkungen des Klimawandels und Herausforderungen

für die medizinische Versorgung in Deutschland

after implementing these systems. However, concerns remain whether the most vulnerable groups such as the elderly and the homeless can be adequately reached in these programs and actions.

\subsection{Einleitung}

Der Klimawandel verändert die globalen Landund Meerestemperaturen. Infolgedessen sind Temperaturschwankungen und extreme Hitzeereignisse in vielen Teilen der Welt häufiger geworden, wie unter anderem die Hitzesommer der Jahre 2003, 2006, 2018 und 2019 zeigen. Seitdem nimmt die Exposition der Bevölkerung gegenüber extremer Hitze weltweit zu, was zu zusätzlichen 220 Millionen Belastungsereignissen durch Hitzewellen (definiert als eine Hitzewelle, die von einer Person mit 65 Jahren oder älter erlebt wird) im Jahr $2018 \mathrm{im}$ Vergleich zum Durchschnitt in den Jahren 1986-2005 führte (Watts et al. 2019). Der Klimawandel hat jedoch nicht nur direkte Folgen, sondern beeinflusst andere gesundheitsrelevante Aspekte. So hängen Höhe und Zusammensetzung der Luftschadstoffe unter anderem von Wetterparametern ab. Im Folgenden möchten wir uns mit den Fragen „Was ist bereits bekannt zu gesundheitlichen Auswirkungen von Luftschadstoffen?", „Was bedeutet der Klimawandel für die Zusammensetzung der Luftschadstoffe?“, „Wie wirkt sich das im Zusammenspiel mit sich ändernden Temperaturen auf die menschliche Gesundheit aus?“ und „Welche Präventions- und Schutzmaßnahmen gibt es?" auseinandersetzen.

\subsection{Gesundheitliche Wirkung von Luftschadstoffen}

\subsubsection{Feinstaub}

Feinstaub (PM, particulate matter) besteht aus einem komplexen Gemisch von festen und flüssigen Partikeln, die mit Durchmessern im Bereich von wenigen Mikrometern $(\mu \mathrm{m})$ mit blo- ßem Auge nicht erkennbar sind. Meist werden sie anhand ihres aerodynamischen Durchmessers in verschiedene Größenkategorien unterteilt: $\mathrm{PM}_{2,5}$-Feinstaub enthält Partikel bis etwa 2,5 $\mu \mathrm{m}$, die Größenklasse $\mathrm{PM}_{10}$ enthält zusätzlich größere Partikel bis etwa $10 \mu \mathrm{m}$. Als Ultrafeinstaub bezeichnet man Partikel mit einem Durchmesser bis etwa o, $1 \mu \mathrm{m}$; er ist in den Größenklassen $\mathrm{PM}_{10}$ und $\mathrm{PM}_{2,5}$ mit enthalten, wird aber bei Routinemessungen bisher nicht gesondert erfasst (Schulz et al. 2019a).

Nicht alle Komponenten des Feinstaubs sind gleich gefährlich. Man geht davon aus, dass etwa $20 \%$ hoch toxisch sind, dazu gehören Verbrennungsprodukte aus Kfz-Emissionen, Industrie und Hausbrand. Ammoniakemissionen der Landwirtschaft tragen ebenfalls zur Bildung von Feinstaub in der Atmosphäre bei (Schulz et al. 2019a). Weniger toxische Komponenten bestehen aus aufgewirbeltem Staub oder biologischen Materialen (z.B. Saharastaub).

\subsubsection{Lungenerkrankungen}

Zusammenhänge zwischen Luftschadstoffen und Atemwegserkrankungen werden seit vielen Jahren erforscht. Zum Beispiel wird der Zusammenhang zwischen $\mathrm{PM}_{2,5}$ und Lungenkrebserkrankungen inzwischen als „kausal“ eingestuft (d.h. für diesen Zusammenhang sind ausreichend Studien vorhanden, in denen zufällige Zusammenhänge, Verzerrungen oder andere Störgrößen ausgeschlossen werden können). Die Hauptursache für Lungenkrebs ist und bleibt das inhalative Zigarettenrauchen. Trotzdem gibt es eine Reihe von Studien, die auch nach einer Adjustierung für Zigarettenrauchen noch deutliche, wenn auch kleine Effekte von Luftschadstoffen sehen (Beelen et al. 2008; Lepeule et al. 2012).

$\mathrm{Zu}$ den Atemwegserkrankungen, die durch Luftschadstoffe hervorgerufen oder verstärkt werden können, gehören die chronisch obstruktive Lungenerkrankung (COPD), Asthma 
bronchiale, Atemwegsinfekte, Bronchitis und Pneumonie sowie die interstitielle Lungenerkrankung (ILD) (Schulz et al. 2019a).

Selbst bei Gesunden haben Luftschadstoffe negative Auswirkungen auf die Lungengesundheit, indem sie, häufig vom Einzelnen noch unbemerkt, die Lungenfunktion verschlechtern (Adam et al. 2015; Int Panis et al. 2017). Sie kann vom Arzt durch Messgrößen wie Lungenvolumina und Atemstromstärken mittels Spirometrie erfasst werden. Besonders bei Kindern und Heranwachsenden wurde in Studien ein negativer Einfluss auf die Lungenentwicklung durch verschiedene Außenluftschadstoffe beobachtet (Barone-Adesi et al. 2015; Gehring et al. 2013).

\subsubsection{Kardiovaskuläre Erkrankungen}

Obwohl Luftschadstoffe primär über die Atemwege in den Körper gelangen und damit zunächst ein Gesundheitsrisiko für die Lunge naheliegt, zeigt die Forschung der vergangenen Jahrzehnte, dass das größte attributable Risiko von Luftschadstoffen beim Herz-Kreislauf-System liegt. Die Auswirkungen reichen von kurzfristigen Gesundheitseinschränkungen, über Krankenhauseinweisungen bis hin zu Todesfällen. Diese können akut bei hohen Konzentrationen oder als Konsequenz von Langzeitbelastungen auftreten (WHO 2013).

Zu den wichtigsten europäischen Kurzzeitstudien im Hinblick auf Partikel in der Außenluft zählt die APHEAz-Studie. In dieser Studie mit Daten aus 29 europäischen Städten konnte ein linearer Zusammenhang zwischen einem Anstieg von $\mathrm{PM}_{10}$ und einer erhöhten Mortalität aufgrund von Herz-Kreislauf-Erkrankungen gezeigt werden (Analitis et al. 2006). Darüber hinaus waren erhöhte Partikelkonzentrationen in der Luft mit vermehrten Krankenhausaufnahmen aufgrund kardiovaskulärer Erkrankungen assoziiert (Sunyer et al. 2003). Entsprechende große Studien in den USA lieferten ähnliche Ergebnisse (Samet et al. 200o).
Die mit Abstand umfassendste Kohortenstudie zum Zusammenhang zwischen der LangzeitExposition gegenüber Feinstaub und der Sterblichkeit ist die "American Cancer Society“ (ACS)-Studie (Krewski 2009; Pope et al. 2002). Für einen Anstieg von $\mathrm{PM}_{2,5}$ wurden statistisch signifikant erhöhte relative Risiken für die Gesamtmortalität und kardiopulmonale Todesfälle gefunden. In der niederländischen „Netherlands Cohort Study-AIR"-Studie war das Wohnen an einer stark befahrenen Straße mit erhöhter kardiovaskulärer Sterblichkeit assoziiert (Beelen et al. 2008).

Epidemiologische Studien zu langfristigen Belastungen mit Feinstäuben (Jahre bis Jahrzehnte) zeigen erhebliche Folgen für die menschliche Gesundheit. Dabei sind die $\mathrm{Zu}-$ sammenhänge für die einzelnen kardiovaskulären Endpunkte unterschiedlich gut gesichert. Aus den veröffentlichten Studien lässt sich eine starke Evidenz für kardiovaskuläre Mortalität, Krankenhauseinweisungen, ischämische Herzerkrankungen bzw. Herzinfarkt und Schlaganfall ableiten. Darüber hinaus kann die Evidenz für die kurzfristige Beeinflussung der vegetativen Balance als gesichert betrachtet werden. Für andere Gesundheitsendpunkte wie Herzinsuffizienz und luftschadstoffassoziierte Arrhythmogenese dagegen stellt sich die Evidenz derzeit als heterogen dar. Ein großer Teil der Studien unterstützt einen Zusammenhang zwischen Luftschadstoffen und einem akuten sowie langfristigen Anstieg des Blutdrucks, einer gestörten vaskulären Homöostase mit endothelialer Dysfunktion und einer Progression atherosklerotischer Veränderungen (Schulz et al. 2019b), jedoch nicht alle.

\subsubsection{Mechanismen}

Feinstaub wird mit der Atmung über die Atemwege in die Lunge transportiert. Insbesondere $\mathrm{PM}_{2,5}$-Feinstaub gelangt bis in die kleinsten Atemwege und Lungenbläschen. Ultrafeine Partikel können außerdem über den Blutkreislauf 
II Gesundheitliche Auswirkungen des Klimawandels und Herausforderungen

für die medizinische Versorgung in Deutschland

$\mathrm{zu}$ anderen Organen gelangen. Zusammengefasst können die Partikel-Effekte durch folgende Mechanismen verursacht werden, die - allein oder gemeinsam - das Risiko für Herz-Kreislaufkrankheiten und kardiovaskuläre Ereignisse erhöhen (Brook et al. 2010; Rückerl et al. 2011):

1. Inhalierte Partikel können Entzündungsreaktionen und oxidativen Stress hervorrufen, die schließlich zu systemischen Entzündungen führen. Die Folge können unter anderem eine Störung der Endothelfunktion, die Bildung von Thromben und ein Fortschreiten atherosklerotischer Läsionen sein.

2. Lungengängige Partikel stimulieren Reflex-Rezeptoren auf der Oberfläche der Lungenbläschen und können somit die autonome Kontrolle des Herzens stören, was beispielsweise zu Herzrhythmusstörungen führen kann.

3. Insbesondere ultrafeine Partikel können in den Blutstrom gelangen, wo sie u.a. die Viskosität des Blutes beeinflussen oder zu lokalen Entzündungsreaktionen führen. So kann man im Blut nach Tagen mit hoher Feinstaubkonzentration erhöhte Entzündungswerte und eine verstärkte Gerinnungsneigung messen (Rückerl et al. 2016).

Man geht davon aus, dass direkte Effekte von Partikeln kardiovaskuläre Ereignisse innerhalb von wenigen Stunden auslösen. Daneben gibt es zunehmend Hinweise, dass Partikel die Entstehung und Progression der Arteriosklerose mit befördern, ein möglicher Mechanismus für die beobachteten Langzeiteffekte (Rückerl et al. 2011).

Kurzzeiteffekte bergen womöglich für gesunde Menschen eher kein Risiko, können aber als plausibler Vorläufer von fatalen Ereignissen bei suszeptiblen Patienten angesehen werden, während repetitive Expositionen bzw. eine hohe Langzeitbelastung zur Entwicklung von kardiovaskulären Erkrankungen beitragen können.
Trotz der guten Studienlage konnte bisher keine untere Wirkungsschwelle identifiziert werden. Das heißt, dass selbst bei niedriger Exposition die Wirkungen mit zunehmender Belastung ansteigen. Dies wird zurzeit in drei großen Studien mit jeweils mehreren Millionen Probanden in Europa, den USA und in Kanada untersucht. Erste Ergebnisse aus den USA zeigen eine lineare Expositions-Wirkungs-Beziehung von $\mathrm{PM}_{2,5}$ mit der Sterblichkeit bis hinunter zu einem Jahresmittelwert von $5 \mu \mathrm{g} / \mathrm{m}^{3}$ (Dominici et al. 2019). Das bedeutet: Jede Reduktion der Belastung ist mit einem Gesundheitsgewinn verbunden.

\subsection{Interaktion von Temperatur und Luftschadstoffen: Einfluss auf Morbidität und Mortalität}

\subsubsection{Klimawandel und Luftverschmutzung}

Luftverschmutzung und Klimawandel sind eng miteinander verknüpft. Die Hauptursachen für den Klimawandel - der Abbau und das Verbrennen von fossilen Energieträgern - produzieren auch große Mengen an Luftschadstoffen. Andererseits tragen Luftschadstoffe zum Klimawandel bei, da sie Einfluss auf atmosphärische Prozesse haben. Je nach seiner Zusammensetzung kann Feinstaub eine kühlende oder wärmende Wirkung auf das lokale und globale Klima haben (Schmale u. Kuik 2013). Ruß beispielsweise, einer der Bestandteile von Feinstaub und das Ergebnis der unvollständigen Verbrennung von Kraftstoffen, absorbiert Solar- und Infrarotstrahlen in der Atmosphäre und hat damit eine wärmende Wirkung. Dies gilt auch für bodennahes Ozon.

Die zu erwartenden Klimaänderungen werden die zukünftige Entwicklung der Luftqualität in Deutschland und Europa vermehrt beeinflussen (Hendriks et al. 2016; Schultz et al. 2017). 


\section{Klimawandel und Ozon}

Klimaprojektionen deuten z.B. an, dass die bodennahe Lufttemperatur in Deutschland zum Ende des 21. Jahrhunderts je nach Klimawandelszenario um 1 bis $4^{\circ} \mathrm{C}$ im Vergleich zur Referenzperiode 1971-200o zunehmen könnte (Jacob et al. 2017). Auch die Häufigkeit und Dauer von Hitzewellen in Deutschland wird sich in $\mathrm{Zu}-$ kunft signifikant erhöhen, was bis zum Ende des 21. Jahrhunderts etwa zu einer Verdreifachung der Anzahl von Hitzewellentagen führen könnte (Zacharias u. Koppe 2015). Ein wärmeres Klima, zusammen mit Veränderungen der Niederschlags- und Windmuster, wird bis zum Ende des 21. Jahrhunderts (wahrscheinlich) die mittleren Ozon-Konzentrationen an der Erdoberfläche im Sommer über Europa erhöhen (Doherty et al. 2017; Lacressonnière et al. 2016). Für den Fall, dass der globale Temperaturanstieg nicht auf $2^{\circ} \mathrm{C}$ beschränkt bleibt, sondern $3^{\circ} \mathrm{C}$ erreichen wird, wird für Europa ein signifikanter Anstieg der Ozonkonzentration relativ zum $2^{\circ} \mathrm{C}$-Fall vorausgesagt (Fortems-Cheiney et al. 2017). Auch die Häufigkeit von sogenannten Ozon-Episoden wird zunehmen (Doherty et al. 2017).

\section{Klimawandel und Feinstaub}

Mögliche Auswirkungen des Klimawandels auf die verschiedenen Größenfraktionen von Feinstaub sind bisher noch weniger verstanden. Aufgrund der unterschiedlichen chemischen Zusammensetzung von Partikeln und der Reaktionen dieser Komponenten auf meteorologische Faktoren sind die Auswirkungen der Meteorologie auf die PM-Konzentrationen sehr komplex und erschweren somit Vorhersagen (Mahmud et al. 2010). Die meisten Studien erwarten eine Abnahme von $\mathrm{PM}_{10}$-Konzentrationen (Juda-Rezler et al. 2012; Lacressonnière et al. 2017), die u.a. auf eine Zunahme der Winterniederschläge, eine Zunahme der Luftfeuchtigkeit oder Veränderungen der Windmuster zu- rückzuführen sind. Für $\mathrm{PM}_{2,5}$-Feinstaub wiederum wird in den meisten Studien eine leichte Zunahme der Konzentrationen erwartet (Lacressonnière et al. 2016; Park et al. 2020). In Bezug auf Feinstaub-Komponenten können höhere Temperaturen bzw. eine höhere Luftfeuchtigkeit z.B. sekundäre organische Aerosole und Sulfat-Aerosole erhöhen, aber Nitrate durch erhöhte Volatilität verringern (Cholakian et al. 2019). Die vorliegenden Ergebnisse zeigen, dass die klimabedingten Veränderungen der PM-Konzentrationen in verschiedenen Regionen und Jahreszeiten sehr unterschiedlich ausfallen können (Juda-Rezler et al. 2012; Megaritis et al. 2014).

\subsubsection{Gesundheitliche Wirkung des Zusammenspiels von hoher Lufttemperatur und Luftschadstoffen}

Hohe Lufttemperaturen zusammen mit intensiver Sonneneinstrahlung begünstigen die Bildung bodennaher Ozonkonzentration. Zudem kann sich die Feinstaubbelastung durch Entstehung von sogenannten sekundären Aerosolen erhöhen (Augustin et al. 2017). Außerdem besteht an heißen Tagen eine geringe Luftzirkulation, daher können vor allem in den Städten erzeugte Luftschadstoffe nicht abgeführt werden und verbleiben in höherer Konzentration in der Luft (Doherty et al. 2017). Trotzdem wurden gesundheitliche Effekte von Wetterveränderungen sowie Luftqualität lange Zeit isoliert betrachtet.

\section{Wechselwirkungen von kurzzeitiger Exposition gegenüber hohen Temperaturen und Ozon oder Feinstaub auf die Mortalität}

So wurde zwar in epidemiologischen Studien zu akuten gesundheitlichen Auswirkungen von Luftschadstoffen in der Regel für Lufttemperatur als Störgröße adjustiert, eine mögliche Wirkungsänderung der Luftschadstoffe auf die 
II Gesundheitliche Auswirkungen des Klimawandels und Herausforderungen

für die medizinische Versorgung in Deutschland

Mortalität durch die Lufttemperatur wurde aber häufig vernachlässigt (Li et al. 2017; Pickford et al. 2020). Die Mehrzahl der bislang veröffentlichten Studien konnte zeigen, dass hohe Temperaturen die Auswirkungen von Ozon oder Feinstaub auf die (ursachenspezifische) Mortalität verstärken (Li et al. 2017; Lou et al. 2019; Shi et al. 2020). Allerdings weisen einige Studien auch auf stärkere Effekte von Ozon und Feinstaub bei gleichzeitig niedrigeren Temperaturen hin bzw. zeigen keine Wirkungsänderung der Luftschadstoffe durch Temperatur (Lou et al. 2019).

Andersherum ist auch eine Wirkungsänderung der Lufttemperatur auf die Mortalität durch Luftschadstoffe möglich (Breitner et al. 2014; Lou et al. 2019; Scortichini et al. 2018). So konnte im europaweiten Projekt EuroHEAT gezeigt werden, dass das Sterberisiko durch Hitze durch gleichzeitig erhöhte Konzentrationen von Ozon und Feinstaub verstärkt wird (Analitis et al. 2014). Ältere Menschen sind in solchen Situationen besonders gefährdet.

In einer weiteren europäischen Studie konnte zudem gezeigt werden, dass sowohl hohe Lufttemperaturen die Auswirkungen von Luftschadstoffen auf die Sterblichkeit an HerzKreislauf- und nicht-unfallbedingten Todesursachen modifizieren, als auch hohe Konzentrationen von Feinstaub, ultrafeinen Partikeln und Ozon den Effekt der Lufttemperatur verstärken (Chen et al. 2018).

\section{Wechselwirkungen von kurzzeitiger Exposition gegenüber Lufttemperatur und Ozon oder Feinstaub auf die Morbidität}

Es gibt bislang nur sehr wenige Studien, die interaktive Effekte bzw. Wirkungsänderungen auf Krankenhauseinweisungen oder andere Morbiditätsendpunkte untersuchten. So zeigten z.B. Studien aus Australien und China, dass sowohl hohe $\mathrm{PM}_{10}$-Konzentrationen die Effekte von hohen Temperaturen auf kardiorespiratorische Krankenhauseinweisungen verstärkten
(Ren et al. 2006), als auch hohe Temperaturen die Auswirkungen von Feinstaub beeinflussten - bei hoher Temperatur waren die PartikelEffekte allgemein stärker (Huang et al. 2016; Ren u. Tong 2006). Auch für $\mathrm{PM}_{2,5}$-Feinstaub war das Risiko für respiratorische Krankenhauseinweisungen in Neuengland, USA, gerade an heißen Tagen erhöht (Yitshak-Sade et al. 2018).

Lepeule et al. (2018) untersuchten die Auswirkungen eines Temperaturanstiegs auf die Lungenfunktion in einer älteren Kohorte von Männern und fanden Hinweise auf eine mögliche Wechselwirkung durch Ruß (Lepeule et al. 2018). Darüber hinaus wurden in dieser Kohorte die Assoziationen zwischen Temperatur und Herzrate und Herzratenvariabilität bei gleichzeitig hohen Ozonkonzentrationen stärker (Ren et al. 2011). Weitere Studien fanden zudem Hinweise auf interaktive Effekte von Temperatur und Feinstaub bzw. Ozon auf Blutdruck (Wu et al. 2015) und Marker für die Endothelfunktion (Lanzinger et al. 2014).

\section{Langzeit-Assoziationen zwischen Feinstaub und Mortalität und Wirkungsänderung durch Temperatur}

$\mathrm{Zu}$ längerfristigen (chronischen) gesundheitlichen Wirkungen des Zusammenspiels von Langzeitbelastung durch Luftschadstoffe und Lufttemperatur gibt es bislang nur sehr wenige Studien. Angesichts des sich wandelnden Klimas ist es aber von Bedeutung, auch die längerfristigen Auswirkungen, wie jährliche Durchschnittstemperaturen, und ihr Zusammenspiel mit einer chronischen Luftschadstoffbelastung zu verstehen.

In einer Studie zur Assoziation zwischen chronischer Feinstaubbelastung und Mortalität in 207 amerikanischen Städten zeigte sich, dass die $\mathrm{PM}_{2,5}$-Effekte in jenen Städten besonders ausgeprägt waren, in denen es im Jahresdurchschnitt wärmer war (Kioumourtzoglou et al. 2016). Ähnliche Effekte wurden in weiteren US- 
amerikanischen Studien beschrieben (Lim et al. 2017; Wang et al. 2017).

\section{Projektionen von gesundheitlichen Auswirkun- gen im Zusammenhang mit Luftverschmutzung und Temperaturänderungen}

Die Vorhersage der gesundheitlichen Auswirkungen zukünftiger Luftverschmutzung unter dem Klimawandel konzentrierte sich bislang hauptsächlich auf Ozon und in geringerem Ausmaß auf $\mathrm{PM}_{2,5}$ (Lou et al. 2019; Orru et al. 2017). Die Ergebnisse deuten darauf hin, dass durch den Klimawandel die mit Luftschadstoffen verbundenen gesundheitlichen Auswirkungen weiter verstärkt werden. Zwar könnte es bis zum Ende des 21. Jahrhunderts aufgrund von Emissionsminderungen einen Rückgang in der vorhergesagten Mortalität geben (Geels et al. 2015; Silva et al. 2017), dieser wird jedoch aufgrund der Bevölkerungsalterung und dem damit verbundenen höheren Anteil an suszeptiblen Personen aufgewogen werden (Lee et al. 2017). Jedoch verwendete bislang nur eine Studie interaktive Effekte von Temperatur und Ozon für die Projektionen (Knowlton et al. 2008).

\subsection{Präventions- und Schutzmaßnahmen}

Eine große Anzahl von Publikationen zeigt, dass die Wirkung von Hitze und Hitzewellen zwischen Populationen und über verschiedene Bevölkerungsuntergruppen hinweg sehr heterogen ausfällt. Die Hauptdeterminanten dieser unterschiedlichen Verläufe sind zum einen das Expositionsniveau (z.B. Häufigkeit, Dauer und Intensität des Wetterereignisses) und zum anderen die Empfindlichkeit (Suszeptibilität) der Bevölkerung sowie ihre Anpassungsfähigkeit (Field et al. 2012). Insbesondere hängt die Hitzeempfindlichkeit von Personen von der Effizienz ihres thermoregulatorischen Systems ab. Dieses ist z.B. bei Säuglingen, Schwangeren und
Menschen mit chronischen Krankheiten und Behinderungen beeinträchtigt, insbesondere aber bei älteren Menschen und bei Menschen mit niedrigem sozioökonomischen Status bzw. schwacher sozialer Unterstützung. In den Industrieländern, also auch in Deutschland, wird die fortschreitende Alterung der Bevölkerung und die zunehmende Verbreitung chronischer Krankheiten den Anteil der gefährdeten Bevölkerung im Zusammenhang mit Hitzewellen infolgedessen erhöhen (McMichael et al. 2008).

Präventions- und Schutzmaßnahmen beeinflussen direkt und indirekt das Bewusstsein, die Bereitschaft, die Wachsamkeit sowie die Suszeptibilität und Adaptationsfähigkeit gegenüber Hitze auf individueller und gemeinschaftlicher Ebene. Viele Städte entwickelten bereits Hitze-Aktionspläne zum Risikomanagement (Harlan u. Ruddell 2011), die Strategien wie Warnsysteme, Echtzeitüberwachungssysteme und vor allem die Identifizierung besonders gefährdeter bzw. schutzbedürftiger Personen in der Bevölkerung beinhalten. Maßnahmen zur Anpassung an den Klimawandel haben somit zudem das Potenzial, gesellschaftliche Ungleichheiten abzubauen (Boeckmann u. Zeeb 2014).

Man unterscheidet dabei Maßnahmen (Michelozzi et al. 2014), die

- das Bewusstsein und die Bereitschaft beeinflussen, z.B. Informationskampagnen und Bildungsprogramme,

- die Suszeptibilität beeinflussen, z.B. Bereitstellung und Infrastruktur von Gesundheitsund Sozialdiensten, oder

- die Anpassungsfähigkeit beeinflussen, z.B. Arbeitsplatzinterventionen, Interventionen zur Kühlung, langfristige Eingriffe in den Städten (z.B. Verbesserung der Wohnqualität oder des städtischen Mikroklimas).

Diese Maßnahmen können dabei auf sehr unterschiedlichen Ebenen ablaufen, wie z.B. in den Gemeinden, in der ärztlichen Crundversorgung, in der Heimpflege, in der Krankenhauspflege und in der Sozialhilfe. 
II Gesundheitliche Auswirkungen des Klimawandels und Herausforderungen

für die medizinische Versorgung in Deutschland

Externe Faktoren wie sozioökonomische Bedingungen, demografische Trends, Klimawandel und Veränderungen in den Luftschadstoffen können Störfaktoren oder Modifikatoren der Wirkweise dieser Maßnahmen sein. Darüber hinaus wird bei einigen Maßnahmen das kurzfristig positive Ergebnis langfristig durch einen negativen Effekt „,ausgeglichen“. So kann der positive Effekt, der sich aus dem Einsatz von Klimaanlagen (siehe Interventionen zur Kühlung, s. Tab. 1) ergibt (Barreca et al. 2016), durch den negativen Effekt aufgewogen werden, dass Klimaanlagen auch zu Emissionen beitragen, die wiederum den Klimawandel beschleunigen (Farbotko u. Waitt 2011). Dies ist der Grund, warum im Kontext des Klimawandels nicht nur Anpassungsmaßnahmen, sondern auch Minderungsmaßnahmen zur Verringerung der Treibhausgasemissionen eingeführt werden sollten.

Einschränkungen und Hindernisse für die Sicherung der öffentlichen Gesundheit bestehen in der Unsicherheit hinsichtlich des zukünftigen Klimas und der sozioökonomischen Bedingungen sowie finanzieller, technologischer, institutioneller, sozialer und individueller kognitiver Grenzen. Insofern müssen jeweils die gesundheitlichen Vorteile von Minderungsstrategien ermittelt und kosteneffiziente Anpassungsmöglichkeiten für die öffentliche Gesundheit bewertet werden (Huang et al. 2011). Studien, die auf Klimaprojektionen basieren, weisen auf einen erheblichen Anstieg der hitzebedingten Mortalität und Morbidität in der Zukunft hin, während Beobachtungsstudien, die auf historischen Klima- und Gesundheitsdaten basieren, eine Abnahme der negativen Auswirkungen während der jüngsten Erwärmung zeigen (Hondula et al. 2015). Die Diskrepanz zwischen den beiden Studiengruppen besteht im Allgemeinen darin, wie gut und wie schnell sich Menschen an Klimaveränderungen anpassen können und wie Anpassungen quantifiziert werden.

In mehreren Studien wurde zudem festgestellt, dass nach der Einführung von Hitze- warnsystemen weniger Menschen an übermäßiger Hitze starben und auch die Nachfrage nach Krankenwägen zurückging (Toloo et al. 2013). In Studien zur Wahrnehmung des öffentlichen Risikos waren sich die meisten Befragten bewusst, wann eine extreme Hitzeepisode auftrat. Sie änderten jedoch nicht unbedingt ihre Verhalten, hauptsächlich aufgrund mangelnder Selbstwahrnehmung als schutzbedürftige Person und Verwirrung in Bezug auf die geeignetsten Maßnahmen. Somit besteht weiterhin Besorgnis darüber, ob die am stärksten gefährdeten Gruppen wie ältere Menschen und Obdachlose in diesen Programmen und Aktionen angemessen erreicht werden können (Bassil u. Cole 2010).

Tabelle 1 stellt die Zielgruppen und Empfehlungen nach Lowe et al. (2011) dar.

In Bezug auf negative Luftschadstoffeinflüsse können bestimmte Verhaltensweisen gesundheitliche Schäden mindern. So ist es förderlich, Lunge und Herz-Kreislauf-System durch regelmäßige körperliche Betätigung (z.B. Radfahren oder Gehen) gesund zu erhalten. Die Ergebnisse von Studien, die die positiven Aspekte der körperlichen Aktivität gegenüber den negativen Aspekten der höheren Luftschadstoff-Exposition abwägen, zeigen bei gesunden Erwachsenen positive Auswirkungen auf die Lungenfunktion, selbst in einer stärker mit Luftschadstoffen belasteten Umgebung (Kubesch et al. 2015), wobei sich die positiven Effekte mit zunehmendem Gehalt an Ruß in der Außenluft verringerten (Laeremans et al. 2018). Auch für kardiovaskuläre Erkrankungen, Krebserkrankungen oder Demenz wird ein Überwiegen der positiven Effekte prognostiziert (Götschi et al. 2015). Zudem bringt ein Wechsel auf aktiven Transport (Radfahren oder Gehen) weitere Co-Benefits wie eine Reduzierung der Treibhausgasemissionen und eine Verbesserung der Luftqualität, was auf lange Sicht der Gesundheit der gesamten Bevölkerung zugute kommt.

Da Partikel oxidativen Stress sowie Entzündungsreaktionen auslösen können (Schulz et 
Tab. 1 Handlungsebenen und empfohlene Aktivitäten (nach Lowe et al. 2011)

\begin{tabular}{|c|c|}
\hline $\begin{array}{l}\text { Handlungsebene/ } \\
\text { Zielgruppe }\end{array}$ & Aktivitäten/Empfehlungen \\
\hline Individuum & $\begin{array}{l}\text { Hitzevermeidung, Begrenzen von (körperlichen) Outdoor-Aktivitäten, Tragen von lockerer und } \\
\text { heller Kleidung, Flüssigkeitszufuhr, Kühlung der Wohn- und Schlafräume, Abkühlung des } \\
\text { Körpers (z.B. durch Aufenthalt in klimatisierten Räumen/Gebäuden), Hilfe/Unterstützung für } \\
\text { schutzbedürftige Personen, Aufsuchen von Beratungsstellen bei gesundheitlichen Problemen, } \\
\text { Aufsuchen von Beratung zur Änderung des Medikamentengebrauchs, Beratung zur Lagerung } \\
\text { von Medikamenten, Beachten von Regeln zur Zubereitung von Lebensmitteln, Zufuhr von } \\
\text { Elektrolyten, Schutz vor Sonnenbrand, Information über prognostizierte Außentemperatur, } \\
\text { Überwachung der Raumtemperatur, Verlagerung von Outdoor-Aktivitäten eher auf kühlere } \\
\text { Stunden/Nacht, Einhaltung vernünftiger Arbeitszeiten, Aufenthalt im Schatten, Abschaltung } \\
\text { nicht verwendeter elektrischer Geräte }\end{array}$ \\
\hline $\begin{array}{l}\text { Akteure des Gesund- } \\
\text { heitswesens } \\
\text { (z.B. Pflegepersonal, } \\
\text { Ärzte), Einrichtungen } \\
\text { der Gesundheits- } \\
\text { versorgung }\end{array}$ & $\begin{array}{l}\text { Identifizierung und Überwachung von gefährdeten Personen, Bereitstellung von Klimaanla- } \\
\text { gen oder gemeinschaftlichen Kühlräumen, Installation von Thermometern zur Überwachung } \\
\text { der Raumtemperatur, häufiger Wechsel der Bettwäsche, angemessene Lagerung von } \\
\text { Nahrungsmitteln, angemessene Flüssigkeitszufuhr, Vermeidung übermäßiger Abkühlung } \\
\left(<28^{\circ} \mathrm{C}\right) \text {, Vermeidung von Kunststoffkontinenzhosen bzw. -polstern, regelmäßiges Wiegen } \\
\text { (Gewichtsverlust ist ein Maß für Dehydration), Gespräch über Anpassungen der Medikamente } \\
\text { mit dem Hausarzt vor der Hitzewelle, Verlagerung von Physiotherapieplänen auf außerhalb } \\
\text { von } 11-16 \text { Uhr, Zusammenarbeit mit der Familie und den entsprechenden Behörden am } \\
\text { Wohnort der schutzbedürftigen Personen } \\
\text { strukturelle Maßnahmen: Beschattung, Anlage von Begrünung, Wasserdächern, Gräsern und } \\
\text { Vegetation }\end{array}$ \\
\hline $\begin{array}{l}\text { öffentliche Ebene: } \\
\text { Gemeinde, Stadt, } \\
\text { Kommune }\end{array}$ & $\begin{array}{l}\text { Auflistung, Angebot und Überwachung öffentlicher Kühlbereiche, Pflege von Listen mit } \\
\text { schutzbedürftigen Personen, Überwachung gefährdeter Personen, Kontaktaufnahme zu } \\
\text { identifizierten gefährdeten Personen, Erkennung der Schutz- bzw. Wasserbedürfnisse von } \\
\text { Obdachlosen, Unterstützung des Transports gefährdeter Personen, Bereitstellung einer } \\
\text { Hotline für gesundheitliche Fragen und Beratung, Entwicklung bzw. Bereitstellung eines } \\
\text { Evakuierungsplans, Vorbereitung auf Stromausfälle }\end{array}$ \\
\hline
\end{tabular}

al. 2019b), ist denkbar, dass z.B. Omega-3-Fettsäuren oxidative Schäden begrenzen. Ein aktueller Review (Barthelemy et al. 2020) zeigte klare Evidenz dafür, dass eine Aufnahme von Antioxidantien über erhöhten Obst- und Gemüsekonsum die mit Luftschadstoffen verbundenen Auswirkungen auf die Gesundheit mildert. Hingegen fanden die Autoren widersprüchliche Belege für antioxidative Nahrungsergänzungsmittel, einschließlich Fischöl, Olivenöl und Vitamin-C- und -E-Zusätze.

Das Zusammenspiel von Hitze und Luftschadstoffen macht es dem Einzelnen oft schwerer, sich in der jeweiligen Situation für die bestmöglichen präventiven Maßnahmen zu entscheiden. Zum Beispiel sollte bei Hitze hauptsächlich in den kühleren Morgen- und Abendstunden gelüftet werden, wohingegen man bei hohen Luftschadstoffen darauf achten muss, die Lüftungszeit in die verkehrsarmen Stunden zu legen und, wenn möglich, nicht unbedingt die Fenster zur Hauptstraße zu öffnen. Auch sollte bei Hitze auf belastende körperliche Aktivität verzichtet werden, was wiederum im möglichen Gegensatz zur oben genannten Empfehlung zur Minderung der negativen Luftschadstoffeffekte durch regelmäßige körperliche Aktivität steht. Auch die 
Klimaanlage als Ausweg bei starker Hitzebelastung bringt gleichzeitig weitere Emissionen mit sich, die wiederum die Luftqualität verschlechtern und den Klimawandel befördern. Daher gilt es, diejenigen Maßnahmen herauszufiltern, die nicht nur einen Aspekt der Umweltbelastungen berücksichtigen, sondern auch Benefits für gleichzeitig bestehende Umweltrisiken bringen.

\section{Literatur}

Adam M, Schikowski T, Carsin AE, Cai Y, Jacquemin B, Sanchez M, et al. (2015) Adult lung function and long-term air pollution exposure. ESCAPE: a multicentre cohort study and meta-analysis. Eur Respir | 45, 38-50

Analitis A, Katsouyanni K, Dimakopoulou K, Samoli E, Nikoloulopoulos AK, Petasakis Y, et al. (2006) Short-term effects of ambient particles on cardiovascular and respiratory mortality. Epidemiology 17, 230-3

Analitis A, Michelozzi P, D'Ippoliti D, De'Donato F, Menne B, Matthies F, et al. (2014) Effects of heat waves on mortality: effect modification and confounding by air pollutants. Epidemiology $25,15-22$

Augustin |, Sauerborn R, Burkart K, Endlicher W, Jochner S, Koppe C (2017) Gesundheit. In: Brasseur G, Jacob D, Schuck-Zöller S (eds) Klimawandel in Deutschland. Springer Spektrum Berlin, Heidelberg

Barone-Adesi F, Dent JE, Dajnak D, Beevers S, Anderson HR, Kelly F), et al. (2015) Long-Term Exposure to Primary Traffic Pollutants and Lung Function in Children: Cross-Sectional Study and Meta-Analysis. PLoS One 10, e0142565

Barreca A, Clay K, Deschenes 0, Greenstone M, Shapiro IS (2016) Adapting to climate change: The remarkable decline in the US temperature-mortality relationship over the twentieth century. I Polit Econ 124, 105-159

Barthelemy I, Sanchez K, Miller MR, Khreis H (2020) New Opportunities to Mitigate the Burden of Disease Caused by Traffic Related Air Pollution: Antioxidant-Rich Diets and Supplements. Int I Environ Res Public Health 17, 630

Bassil KL, Cole DC (2010) Effectiveness of public health interventions in reducing morbidity and mortality during heat episodes: a structured review. Int | Environ Res Public Health 7, 991-1001

Beelen R, Hoek G, van den Brandt PA, Goldbohm RA, Fischer P, Schouten LI, et al. (2008) Long-term effects of traffic-related air pollution on mortality in a Dutch cohort (NLCS-AIR study). Environ Health Perspect 116, 196-202

Boeckmann M, Zeeb H (2014) Using a social justice and health framework to assess European climate change adaptation strategies. Int | Environ Res Public Health 11, 12389-411

Breitner S, Wolf K, Devlin RB, Diaz-Sanchez D, Peters A, Schneider A (2014) Short-term effects of air temperature on mortality and effect modification by air pollution in three cities of $\mathrm{Ba}$ varia, Germany: a time-series analysis. Sci Total Environ 485486, 49-61

Brook RD, Rajagopalan S, Pope CA, 3rd, Brook JR, Bhatnagar A, Diez-Roux AV, et al. (2010) Particulate matter air pollution and cardiovascular disease: An update to the scientific statement from the American Heart Association. Circulation 121, 233178

Chen K, Wolf K, Breitner S, Gasparrini A, Stafoggia M, Samoli E, et al. (2018) Two-way effect modifications of air pollution and air temperature on total natural and cardiovascular mortality in eight European urban areas. Environ Int 116, 186-196

Cholakian A, Colette A, Coll I, Ciarelli G, Beekmann M (2019) Future climatic drivers and their effect on PM10 components in Europe and the Mediterranean Sea. Atmos Chem Phys 19, 44594484

Doherty RM, Heal MR, O'Connor FM (2017) Climate change impacts on human health over Europe through its effect on air quality. Environ Health 16, 118

Dominici F, Schwartz |, Di Q, Braun D, Choirat C, Zanobetti A (2019) Assessing Adverse Health Effects of Long-Term Exposure to Low Levels of Ambient Air Pollution: Phase 1. Res Rep Health Eff Inst 200, 1-51

Farbotko C, Waitt G (2011) Residential air-conditioning and climate change: voices of the vulnerable. Health Promot | Aus$\operatorname{tr} 22,13-15$

Field CB, Barros V, Stocker TF, Dahe Q (2012) Managing the risks of extreme events and disasters to advance climate change adaptation: special report of the intergovernmental panel on climate change, Cambridge University Press

Fortems-Cheiney A, Foret G, Siour G, Vautard R, Szopa S, Dufour $\mathrm{G}$, et al. (2017) $\mathrm{A} 3^{\circ} \mathrm{C}$ global RCP8.5 emission trajectory cancels benefits of European emission reductions on air quality. $\mathrm{Na}$ ture Communications 8,89

Geels C, Andersson C, Hänninen 0, Lansø AS, Schwarze PE, Skjøth CA, Brandt I (2015) Future premature mortality due to 03, secondary inorganic aerosols and primary PM in Europesensitivity to changes in climate, anthropogenic emissions, population and building stock. Int I Environ Res Public health 12, 2837-2869

Gehring U, Gruzieva 0, Agius RM, Beelen R, Custovic A, Cyrys I, et al. (2013) Air pollution exposure and lung function in children: the ESCAPE project. Environ Health Perspect 121, 135764

Götschi T, Tainio M, Maizlish N, Schwanen T, Goodman A, Woodcock I (2015) Contrasts in active transport behaviour across four countries: how do they translate into public health benefits? Prev Med 74, 42-8

Harlan SL, Ruddell DM (2011) Climate change and health in cities: impacts of heat and air pollution and potential co-benefits from mitigation and adaptation. Curr Opin Environ Sustain 3, 126-134

Hendriks C, Forsell N, Kiesewetter G, Schaap M, Schöpp W (2016) Ozone concentrations and damage for realistic future European climate and air quality scenarios. Atmos Environ 144, 208-219 
Hondula DM, Balling RC, Vanos JK, Georgescu M (2015) Rising temperatures, human health, and the role of adaptation. Curr Clim Change Rep 1, 144-154

Huang C, Vaneckova P, Wang X, Fitzgerald G, Guo Y, Tong S (2011) Constraints and barriers to public health adaptation to climate change: a review of the literature. Am J Prev Med 40, 183-90

Huang F, Luo Y, Guo Y, Tao L, Xu Q, Wang C, et al. (2016) Particulate Matter and Hospital Admissions for Stroke in Beijing, China: Modification Effects by Ambient Temperature. I Am Heart AsSoc 5, e003437

Int Panis L, Provost EB, Cox B, Louwies T, Laeremans M, Standaert A, et al. (2017) Short-term air pollution exposure decreases lung function: a repeated measures study in healthy adults. Environ Health 16, 60

Jacob D, Kottmeier C, Petersen I, Rechid D, Teichmann C (2017) Regionale Klimamodellierung. In: Brasseur G, Jacob D, SchuckZöller S (eds) Klimawandel in Deutschland. Springer Spektrum Berlin, Heidelberg

Juda-Rezler K, Reizer M, Huszar P, Krüger BC, Zanis P, Syrakov D, et al. (2012) Modelling the effects of climate change on air quality over Central and Eastern Europe: concept, evaluation and projections. Clim Res 53, 179-203

Kioumourtzoglou M-A, Schwartz |, James P, Dominici F, Zanobetti A (2016) PM2.5 and Mortality in 207 US Cities: Modification by Temperature and City Characteristics. Epidemiology 27, 221-227

Knowlton K, Hogrefe C, Lynn B, Rosenzweig C, Rosenthal ), Kinney PL (2008) Impacts of Heat and Ozone on Mortality Risk in the New York City Metropolitan Region Under a Changing Climate. Seasonal Forecasts, Climatic Change and Human Health 143-160

Krewski D (2009) Evaluating the effects of ambient air pollution on life expectancy. N Engl I Med 360, 413-5

Kubesch NI, de Nazelle A, Westerdahl D, Martinez D, CarrascoTurigas G, Bouso L, et al. (2015) Respiratory and inflammatory responses to short-term exposure to traffic-related air pollution with and without moderate physical activity. Occup Environ Med 72, 284-93

Lacressonnière G, Foret G, Beekmann M, Siour G, Engardt M, Gauss $M$, et al. (2016) Impacts of regional climate change on air quality projections and associated uncertainties. Clim Change 136, 309-324

Lacressonnière $\mathrm{G}$, Watson L, Gauss $\mathrm{M}$, Engardt $\mathrm{M}$, Andersson C, Beekmann M, et al. (2017) Particulate matter air pollution in Europe in $\mathrm{a}+2^{\circ} \mathrm{C}$ warming world. Atmos Environ 154, 129-140

Laeremans M, Dons E, Avila-Palencia I, Carrasco-Turigas G, Orjuela-Mendoza IP, Anaya-Boig E, et al. (2018) Black Carbon Reduces the Beneficial Effect of Physical Activity on Lung Function. Med Sci Sports Exerc 50, 1875-1881

Lanzinger S, Breitner S, Neas L, Cascio W, Diaz-Sanchez D, Hinderliter A, et al. (2014) The impact of decreases in air temperature and increases in ozone on markers of endothelial function in individuals having type-2 diabetes. Environ Res 134, 331-338
Lee JY, Lee SH, Hong S-C, Kim H (2017) Projecting future summer mortality due to ambient ozone concentration and temperature changes. Atmos Environ 156, 88-94

Lepeule I, Laden F, Dockery D, Schwartz I (2012) Chronic exposure to fine particles and mortality: an extended follow-up of the Harvard Six Cities study from 1974 to 2009. Environ Health Perspect 120, 965-70

Lepeule I, Litonjua AA, Gasparrini A, Koutrakis P, Sparrow D, Vokonas PS, Schwartz I (2018) Lung function association with outdoor temperature and relative humidity and its interaction with air pollution in the elderly. Environ Res 165, 110-117

Li I, Woodward A, Hou XY, Zhu T, Zhang I, Brown H, et al. (2017) Modification of the effects of air pollutants on mortality by temperature: A systematic review and meta-analysis. Sci Total Environ 575, 1556-1570

Lim C, Yinon L, Ahn I, Hayes R, Shao Y, Thurston GD (2017) Effect Modification of Long-Term PM2.5-Mortality Relationship by Temperature in the US. American Thoracic Society 2017 International Conference abstract.

Lou I, Wu Y, Liu P, Kota SH, Huang L (2019) Health Effects of Climate Change Through Temperature and Air Pollution. Curr Pollut Rep 5, 144-158

Lowe D, Ebi KL, Forsberg B (2011) Heatwave early warning systems and adaptation advice to reduce human health consequences of heatwaves. Int I Environ Res Public Health 8, 4623-48

Mahmud A, Hixson M, Hu J, Zhao Z, Chen SH, Kleeman MJ (2010) Climate impact on airborne particulate matter concentrations in California using seven year analysis periods. Atmos Chem Phys 10, 11097-11114

McMichael AJ, Wilkinson P, Kovats RS, Pattenden S, Hajat S, Armstrong B, et al. (2008) International study of temperature, heat and urban mortality: the 'ISOTHURM' project. Int I Epidemiol 37, 1121-31

Megaritis AG, Fountoukis C, Charalampidis PE, Denier van der Gon HAC, Pilinis C, Pandis SN (2014) Linking climate and air quality over Europe: effects of meteorology on $\mathrm{PM}_{2.5}$ concentrations. Atmos Chem Phys 14, 10283-10298

Michelozzi P, Bargagli AM, Vecchi S, De Sario M, Schifano P, Davoli M (2014) Interventions for reducing adverse health effects of high temperature and heatwaves. Cochrane Database of Systematic Reviews

Orru H, Ebi KL, Forsberg B (2017) The Interplay of Climate Change and Air Pollution on Health. Curr Environ Health Rep 4, 504513

Park S, Allen RJ, Lim CH (2020) A likely increase in fine particulate matter and premature mortality under future climate change. Air Qual Atmos Hlth 13, 143-151

Pickford R, Kraus U, Frank U, Breitner S, Markevych I, Schneider A (2020) Kombinierte Effekte verschiedener Umweltfaktoren auf die Gesundheit: Luftschadstoffe, Temperatur, Grünflächen, Pollen und Lärm. Bundesgesundheitsblatt 63, 962-971

Pope CA, 3rd, Burnett RT, Thun MI, Calle EE, Krewski D, Ito K, Thurston GD (2002) Lung cancer, cardiopulmonary mortality, and long-term exposure to fine particulate air pollution. JAMA 287, 1132-41 
Ren C, O'Neill MS, Park SK, Sparrow D, Vokonas P, Schwartz | (2011) Ambient temperature, air pollution, and heart rate variability in an aging population. Am I Epidemiol 173, 1013-21

Ren C, Tong S (2006) Temperature modifies the health effects of particulate matter in Brisbane, Australia. Int | Biometeorol 51, 87-96

Ren C, Williams GM, Tong S (2006) Does particulate matter modify the association between temperature and cardiorespiratory diseases? Environ Health Perspect 114, 1690-6

Rückerl R, Schneider A, Breitner S, Cyrys I, Peters A (2011) Health effects of particulate air pollution: A review of epidemiological evidence. Inhal Toxicol 23, 555-92

Rückerl R, Schneider A, Hampel R, Breitner S, Cyrys |, Kraus U, et al. (2016) Association of novel metrics of particulate matter with vascular markers of inflammation and coagulation in susceptible populations - results from a panel study. Environ Res 150, 337-347

Samet JM, Zeger SL, Dominici F, Curriero F, Coursac I, Dockery DW, et al. (2000) The National Morbidity, Mortality, and Air Pollution Study. Part II: Morbidity and mortality from air pollution in the United States. Res Rep Health Eff Inst 94, 5-70; discussion 71-9

Schmale J, Kuik F (2013) Gefahr für die Gesundheit und Klima: Kurzlebige Klimawirksame Schadstoffe. Institute for Advanced Sustainability Studies (IASS) Potsdam

Schultz M, Klemp D, Wahner A (2017) Luftqualität. In: Brasseur G, Jacob D, Schuck-Zöller S (eds) Klimawandel in Deutschland. Springer Spektrum Berlin, Heidelberg

Schulz H, Karrasch S, Bölke G, Cyrys I, Hornberg C, Pickford R, et al. (2019a) Atmen: Luftschadstoffe und Gesundheit-Teil I. Pneumologie 73, 288-305

Schulz H, Karrasch S, Bölke G, Cyrys I, Hornberg C, Pickford R, et al. (2019b) Atmen: Luftschadstoffe und Gesundheit-Teil III. Pneumologie 73, 407-429

Scortichini M, De Sario M, de'Donato FK, Davoli M, Michelozzi P, Stafoggia M (2018) Short-Term Effects of Heat on Mortality and Effect Modification by Air Pollution in 25 Italian Cities. Int | Environ Res Public Health 15,
Shi W, Sun Q, Du P, Tang S, Chen C, Sun Z, et al. (2020) Modification Effects of Temperature on the Ozone - Mortality Relationship: A Nationwide Multicounty Study in China. Environ Sci Technol 54, 2859-2868

Silva RA, West II, Lamarque J-F, Shindell DT, Collins W|, Faluvegi G, et al. (2017) Future global mortality from changes in air pollution attributable to climate change. Nat Clim Chang 7, 647651

Sunyer I, Ballester F, Tertre AL, Atkinson R, Ayres JG, Forastiere F, et al. (2003) The association of daily sulfur dioxide air pollution levels with hospital admissions for cardiovascular diseases in Europe (The APHEA-II study). Eur Heart I 24, 752-60

Toloo GS, Fitzgerald G, Aitken P, Verrall K, Tong S (2013) Are heat warning systems effective? Environ Health 12, 27

Wang Y, Shi L, Lee M, Liu P, Di Q, Zanobetti A, Schwartz ID (2017) Long-term Exposure to PM2.5 and Mortality Among Older Adults in the Southeastern US. Epidemiology 28, 207-214

Watts N, Amann M, Arnell N, Ayeb-Karlsson S, Belesova K, Boykoff M, et al. (2019) The 2019 report of The Lancet Countdown on health and climate change: ensuring that the health of a child born today is not defined by a changing climate. Lancet 394, 1836-1878

WHO (2013) Review of evidence on health aspects of air pollution - REVIHAAP. Technical Report. WHO Regional Office for Europe: Copenhagen. p. 309.

Wu S, Deng F, Huang I, Wang X, Qin Y, Zheng C, et al. (2015) Does ambient temperature interact with air pollution to alter blood pressure? A repeated-measure study in healthy adults. | Hypertens 33, 2414-21

Yitshak-Sade M, Bobb JF, Schwartz ID, Kloog I, Zanobetti A (2018) The association between short and long-term exposure to PM2.5 and temperature and hospital admissions in New England and the synergistic effect of the short-term exposures. Sci Total Environ 639, 868-875

Zacharias S, Koppe C (2015) Einfluss des Klimawandels auf die Biotropie des Wetters und die Gesundheit bzw. die Leistungsfähigkeit der Bevölkerung in Deutschland, Umweltbundesamt Dessau-Roßlau 


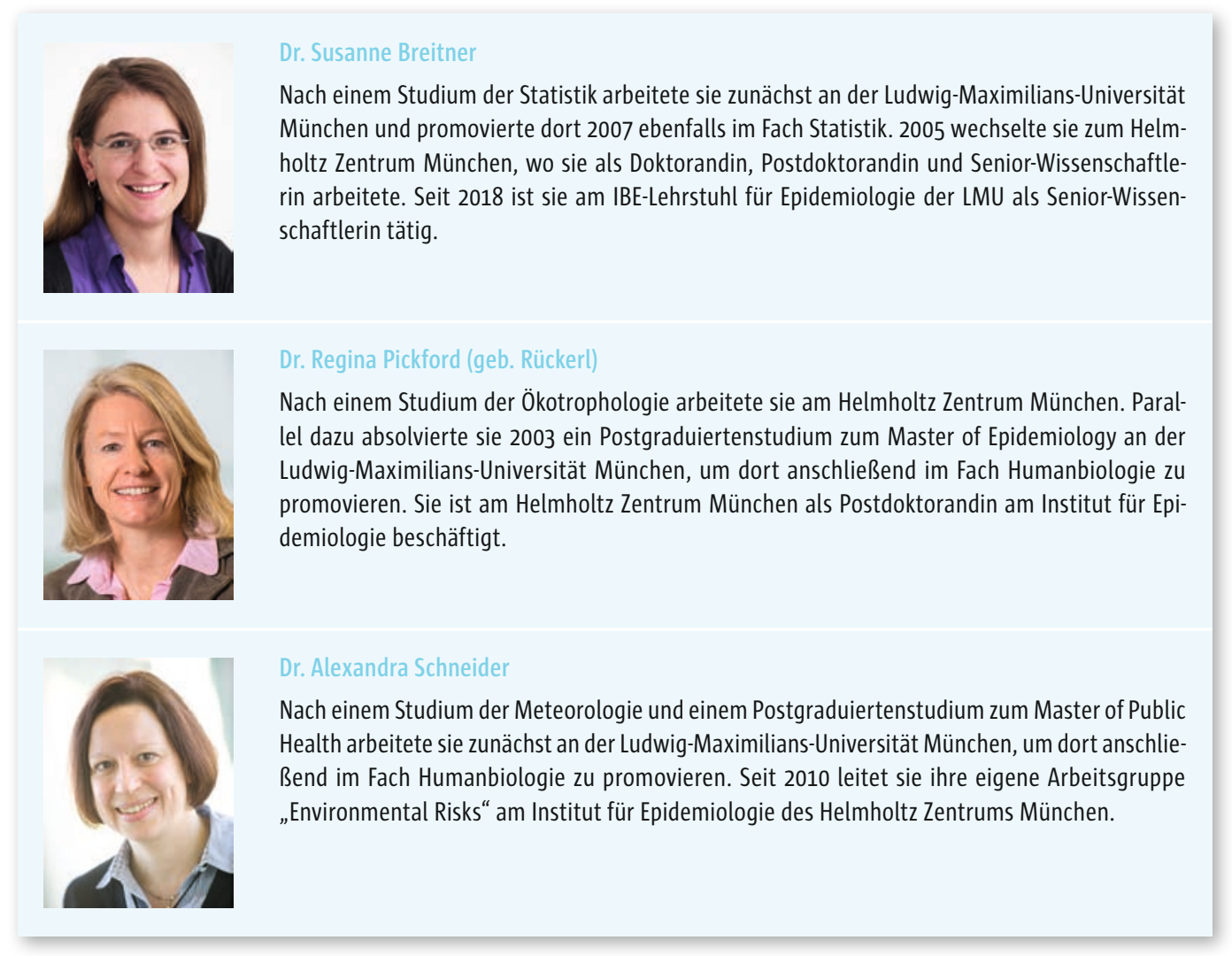

